Published in "Journal of Infection 80(1): 121-142, 2020"

which should be cited to refer to this work.

\section{In-vitro evaluation of a dual carbapenem combination against carbapenemase-producing Acinetobacter baumannii}

Sir,

Russo and colleagues, in this Journal, recently reported a multicenter study of bloodstream infections caused by carbapenemresistant $A$. baumannii (CRAb). ${ }^{1}$ This underlines that the spread of CRAb is becoming a major public health concern, being listed among the top three priorities for multidrug-resistant bacteria of the World Health Organization. ${ }^{2,3}$ It is known that almost all carbapenem-resistant $A$. baumannii produce a carbapenemase. ${ }^{3}$ The types of carbapenemases encountered are mostly specific to the Acinetobacter genus. ${ }^{2,3}$ These are mainly Ambler class D ß-lactamases possessing carbapenemase activity such as the overexpressed and naturally-occurring OXA-51 and the acquired enzymes of the OXA-23-, OXA-40- and OXA-58-types as well as the rare OXA-143. ${ }^{3}$ The OXA-23-type enzymes are the most widespread carbapenemases in $A$. baumannii. ${ }^{3}$ In addition, few other carbapenemases have been identified in A. baumannii, corresponding to the Ambler class A ß-lactamases of the KPC-type, and the Ambler class B metallo- $\beta$-lactamases of the IMP, VIM and NDM types. ${ }^{3}$ The CRAb clinical isolates are in their vast majority resistant to many other important antibiotics such as all ß-lactams, fluoroquinolones and aminoglycosides, making related infections challenging to treat. As opposed to carbapenem-resistant Enterobacterales, the prevalence of CRAb among Acinetobacter sp is quite high, ranging from 40 to $80 \%$,worldwide. ${ }^{4}$

None of the novel ß-lactam/ß-lactamase inhibitor combinations currently available (ceftazidime/avibactam, ceftolozane/ tazobactam, imipenem/relebactam, meropenem/ varbobactam) are efficient for treating infections due to CRAb since none of those antibiotics is active against class D carbapenemase producers. Therefore, very few therapeutic options are available currently for treating MDR A. baumannii, ${ }^{1,5}$ and most of them include antibiotic combinations with polymyxins or tigecycline which efficacy remains debatable. ${ }^{1}$ In addition, polymyxins and tigecycline molecules raise concern of poor diffusion and possible toxicity.

Early studies performed using animal models of infections or experimental treatments of humans indicate that dual-carbapenem combinations may be efficient for treating infections due to KPC and NDM producing Enterobacterales, and particularly Klebsiella pneumoniae. $^{6-10}$ Further in-vitro and in-vivo studies validated those findings. ${ }^{6-9}$ The rationale of using two carbapenems (in particular ertapenem with another carbapenem) would be that ertapenem may bind to the active site of the carbapenemase with high affinity, and therefore may prevent the hydrolysis of the other carbapenem molecule. The mode of action of these dual
Table 1

Evaluation of the efficacy of a dual carbapenem combination against carbapenemase-producing Acinetobacter baumannii.

\begin{tabular}{llllll}
\hline Strain & Country of isolation & Carbapenemases & \multicolumn{2}{l}{ MIC $(\mathrm{mg} / \mathrm{L})$} & \multirow{2}{*}{ FIC } \\
\cline { 4 - 5 } & & & IPM & MEM & \\
\hline R629 & France & OXA-23 & 64 & 128 & 0.50 \\
R625 & France & OXA-23 & 32 & 64 & 0.50 \\
R628 & France & OXA-23 & 32 & 32 & 0.75 \\
R627 & Bahrein & OXA-23 & 32 & 32 & 1 \\
R622 & France & OXA-23 & 64 & 64 & 0.75 \\
R626 & Bahrein & OXA-23 & 32 & 64 & 0.75 \\
R623 & Saudi Arabia & OXA-23 & 32 & 64 & 0.50 \\
R624 & Colombia & OXA-23 & 8 & 64 & 1 \\
N715 & France & OXA-23 & 16 & 16 & 0.75 \\
N688 & Switzerland & OXA-23 & 32 & 32 & 1 \\
N690 & Switzerland & OXA-23 & 64 & 64 & 0.50 \\
R703 & Brazil & OXA-40 & 64 & 64 & 0.60 \\
R704 & France & OXA-40 & 64 & 64 & 0.60 \\
R824 & Turkey & OXA-58 & 32 & 64 & 0.50 \\
R825 & Romania & OXA-58 & 32 & 8 & 0.75 \\
N7 & Switzerland & OXA-72 & 64 & 128 & 0.75 \\
N420 & Switzerland & NDM-1 & 8 & 8 & 0.60 \\
N15 & Switzerland & NDM-1 & 32 & 32 & 0.75 \\
R32 & France & NDM-1 & 32 & 32 & 0.60 \\
R34 & France & NDM-1 & 16 & 64 & 0.50 \\
N655 & Switzerland & NDM-1 & 64 & 64 & 1 \\
N739 & Switzerland & NDM-5 & 128 & 64 & 0.60 \\
R71 & France & IMP-4 & 16 & 32 & 0.75 \\
\hline
\end{tabular}

IPM: Imipenem; MEM: Meropenem.

combinations would be somehow similar to that of amoxicillin or ceftazidime combined with the ß-lactamase inhibitors clavulanic acid or avibactam, respectively.

To the best of our knowledge, dual carbapenem combinations have never been tested against carbapenemase-producing $A$. baumannii. Therefore, our goal was to evaluate the in-vitro activity of a dual carbapenem combination, i.e. imipenem and meropenem, against $A$. baumannii isolates producing different types of carbapenemases. Imipenem and meropenem were retained since those carbapenems are widely used for treating infections caused by $A$. baumannii. Ertapenem was not tested since $A$. baumannii is naturally resistant to ertapenem, likely related to its weak penetration through the outer membrane.

A collection of twenty three non-clonally related $A$. baumannii isolates recovered from clinical samples from Barhein, Brazil, Colombia, France, Saudi Arabia, Switzerland, and Turkey was tested. All the isolates were non susceptible to imipenem and meropenem (Table 1). Following the latest EUCAST breakpoints (http://www.eucast.org/), isolates with MIC values of imipenem and meropenem $\leq 2 \mathrm{mg} / \mathrm{L}$ are categorized as susceptible, whereas those with MICs of imipenem $>4 \mathrm{mg} / \mathrm{L}$ or and MIC of 
meropenem $>8 \mathrm{mg} / \mathrm{L}$ are considered resistant. The nature of the carbapenemase produced by each isolate was characterized at the molecular level. Our collection included eleven OXA-23, two OXA40, two OXA-58, one OXA 72, six NDM and one IMP producers that mirrors the variety of acquired carbapenemases identified in $A$. baumannii and their distribution worldwide (Table 1). Fractional inhibitory concentration (FICs) index were calculated according to the following formula, $\Sigma$ FIC $=$ FIC of imipenem + FIC of meropenem where FIC of imipenem or meropenem=MIC of imipenem or meropenem in combination, divided by the MIC of https://www.ncbi.nlm.nih.gov/pubmed/?term=NDM+egypt imipenem or meropenem alone. Interpretation of the results was based on the following; FIC values of $\leq 0.5$ indicate synergy, FIC values of $>0.5$ to 4 indicate no interaction, and FIC values $>4$ indicate antagonism. ${ }^{11}$

Six out of the twenty three isolates tested showed synergy activity of imipenem and meropenem (ca. 25\%) whereas the combination of imipenem and meropenem were indifferent for the other tested carbapenemase-producing isolates. Among the six isolates for which a synergy was identified, different types of carbapenemases were produced. No antagonism between imipenem and meropenem was identified for any of the isolates.

The rate of in-vitro synergy observed between imipenem and meropenem for those CRAb corresponds to that identified for carbapenemase-producing $K$. pneumoniae. ${ }^{11}$ In addition, we identified here a synergy for a single $A$. baumannii isolate producing NDM-1 whereas that synergy as determined by the results of th FIC values was not identified for any of the NDM-1-producing $K$. pneumoniae tested previously. ${ }^{11}$

Our study suggests that dual carbapenem combination may be effective, to some extent, against some carbapenemase-producing A. baumannii. This dual combination may therefore work in some - cases to inhibit not only the activity of carbapenemases of class A (KPC) and class B (NDM) as known in Enterobacteriales. but also of those peculiar class $D$ carbapenemases specific to $A$. baumannii (OXA-23, OXA-40 and OXA-58). Due to the dearth of novel antibiotics for treating infections due to MDR A. baumannii, this dual carbpenem combination may offer an alternative since all MDR $A$. baumannii produce a carbapenemase. By targeting the inactivation of the carbapenemase, we are targeting actually the MDR pattern of those strains.

Further investigations using animal models of infection and linical trials are required to further establish this treatment as an alternative against MDR A. baumannii.

\section{Funding}

This work was financed by the University of Fribourg and the National Reference Center for Emerging Antibiotic Resistance in Switzerland, Fribourg (Switzerland).

\section{Declaration of Competing Interest}

None to declare.

\section{References}

1. Russo A., Bassetti M., Ceccarelli G., et al. Bloodstream infections caused by carbapenem-resistant Acinetobacter baumannii; clinical features, therapy and outcome from a multicenter study. J Infection 2019;79:130-8.

2. Meletis G.. Carbapenem resistance: overview of the problem and future perspectives. Ther Adv Infect Dis 2016;3:15-21.

3. Potron A., Poirel L., Nordmann P.. Emerging broad-spectrum resistance in Pseudomonas aeruginosa and Acinetobacter baumannii: mechanisms and epidemiology. Int J Antimicrob Agents 2015;45:568-84

4. Eichenberger E.M., Thaden J.. Epidemiology and mechanisms of resistance of extensively drug-resistant bacteria. Antibiotics 2019;8:37. doi:10.3390/ antibiotics8020037.

5. Zhanel G.G., Golden A.R., Zelenitsky S., et al. Cefidericol; a siderophore cephalosporin with activity against carbapenem-resistant and multidrug-resistant Gram-Negative bacilli. Drug 2019;79:271-89.
6. Bulik C.C., Nicolau D.P.. Double-carbapenem therapy for a carbapenemase-producing Klebsiella pneumoniae. Antimicrob Agents Chemother 2011:55:3002-4.

7. Giammarellou H., Galani L., Baziaka F., et al. Effectiveness of a double carbapenem regimen for infections in humans due to carbapenemase -producing pandrug-resistant Klebsiella pneumoniae. Antimicrob Agents Chemother 2013; 57:2388-90.

8. Wiskirchen D.E., Crandon J.L., Nicolau D.P.. Impact of various conditions on the efficacy of dual carbapenem therapy against KPC-producing Klebsiella pneumoniae. Int J Antimicrob Agents 2013;41:582-5.

9. Wiskirchen D.E., Nordmann P., Crandon J.L., et al. Efficacy of humanized carbapenem exposure against New Delhi metallo- ß-lactamase (NDM-1) -producing Enterobacteriaceae in a murine infection model. Antimicrob Agents Chemother 2013;57:3939-40

10. Ceccarelli G., Falcone M., Giordano A., et al. Successful ertapenem-doripenen combination treament of bacteremic ventilator-associated pneumoniae due to colistin-resistant KPC-producing Klebsiella pneumoniae. Antimicrob Agents Chemother 2013:57:2900-1.

11. Poirel L., Kieffer N., Nordmann P.. In vitro evaluation of dual carbapenem combinations against carbapenemase-producing Enterobacteriaceae. J Antimicrob Chemother 2016;71::156-8.

Patrice Nordmann

Emerging Antibiotic Resistance Unit, Medical and Molecular Microbiology, Department of Medicine, University of Fribourg, Fribourg, Switzerland

INSERM European Unit (LEA-IAME, France), University of Fribourg, Fribourg, Switzerland

National Reference Center for Emerging Antibiotic Resistance, University of Fribourg, Fribourg, Switzerland University of Lausanne and University Hospital Center, Lausanne, Switzerland

Julien Perler, Nicolas Kieffer Emerging Antibiotic Resistance Unit, Medical and Molecular Microbiology, Department of Medicine, University of Fribourg, Fribourg, Switzerland

Laurent Poirel

Emerging Antibiotic Resistance Unit, Medical and Molecular Microbiology, Department of Medicine, University of Fribourg, Fribourg, Switzerland INSERM European Unit (LEA-IAME, France), University of Fribourg, Fribourg, Switzerland National Reference Center for Emerging Antibiotic Resistance, University of Fribourg, Fribourg, Switzerland

*Corresponding author at: Medical and Molecular Microbiology Unit, Department of Medicine, University of Fribourg, Chemin du Musée 18, Fribourg CH-1700, Switzerland. E-mail address: patrice.nordmann@unifr.ch (P. Nordmann) 